\title{
Osseous sarcoidosis mimicking metastatic breast cancer
}

\author{
Henry Li BSc, Laurence Stillwater MD, Mark Bryanton MD, Christina A. Kim MD
}

Cite as: CMAJ 2020 July 13;192:E799-802. doi: 10.1503/cmaj.191661

A 49-year-old premenopausal woman underwent lumpectomy and sentinel lymph node biopsy for grade 1 invasive ductal carcinoma of the right breast. The tumour measured $1.6 \mathrm{~cm}$ and was positive for estrogen receptor and progesterone receptor, and negative for human epidermal receptor 2. The surgical margins were negative. One sentinel lymph node was positive, consistent with stage $2 \mathrm{~A}$ ( $\mathrm{pT} 1 \mathrm{cN} 1$ ) disease.

Postoperatively, a staging technetium 99m-methyl diphosphonate (99mTc MDP) whole-body bone scan showed nonspecific MDP-avid lesions in the right iliac bone, right ischium and right proximal humeral metaphysis (Figure 1A). Chestabdomen-pelvis computed tomography (CT) also showed subtle lesions in the thoracic spine. Overall, the bone lesions were not thought to be metastatic, but repeat imaging in 3-6 months was recommended.

Within the chest, there were multiple pulmonary nodules present in a perilymphatic distribution and mildly enlarged bilateral hilar lymph nodes, which raised the possibility of sarcoidosis

\section{KEY POINTS}

- Osseous sarcoidosis is an uncommon manifestation of sarcoidosis that can mimic metastatic bone disease on imaging.

- Both osseous sarcoidosis and bone metastases from breast cancer can present as lytic, blastic or mixed lesions.

- In patients with breast cancer and imaging findings suggestive of skeletal metastases, it is important to keep a broad differential diagnosis and consider bone biopsy for a definitive diagnosis.

- Breast cancer may contribute to the development of sarcoidosis.

(Figure 2). Transbronchial biopsy of the lung was done with a flexible fibre optic bronchoscope, which showed multinucleated giant cell granulomas with no necrosis. There was no evidence of malignant disease. Bronchial washings showed normal respiratory flora, and fungal cultures and stains for acid-fast bacilli gave negative results.
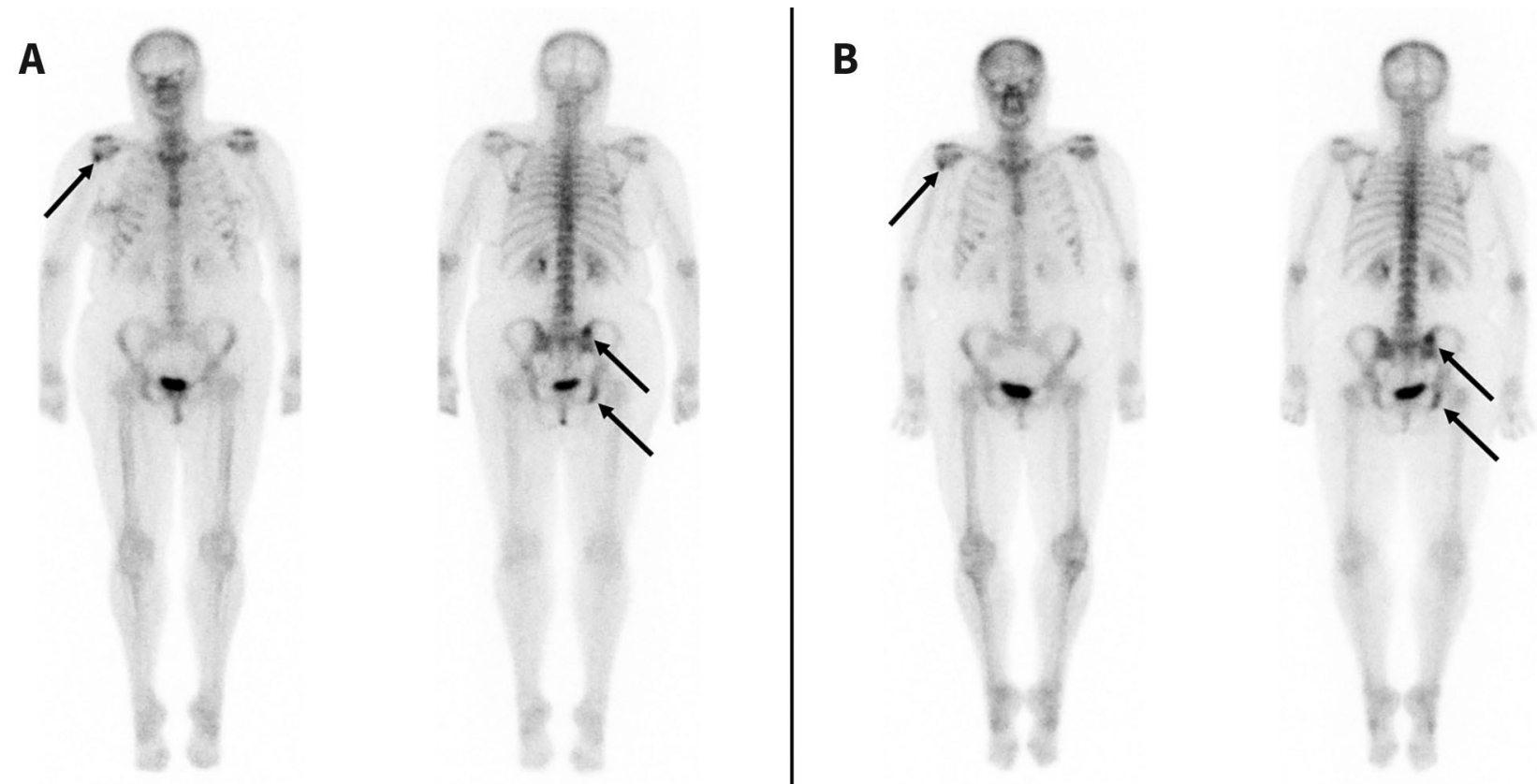

Figure 1: Delayed technetium 99m-methyl diphosphonate (99mTc-MDP) whole-body skeletal scans in a 49-year-old woman with ductal carcinoma of the right breast. (A) MDP-avid lesions in the right iliac bone, right ischium and right proximal humeral metaphysis (arrows). (B) Two years later, the lesions were essentially unchanged. 


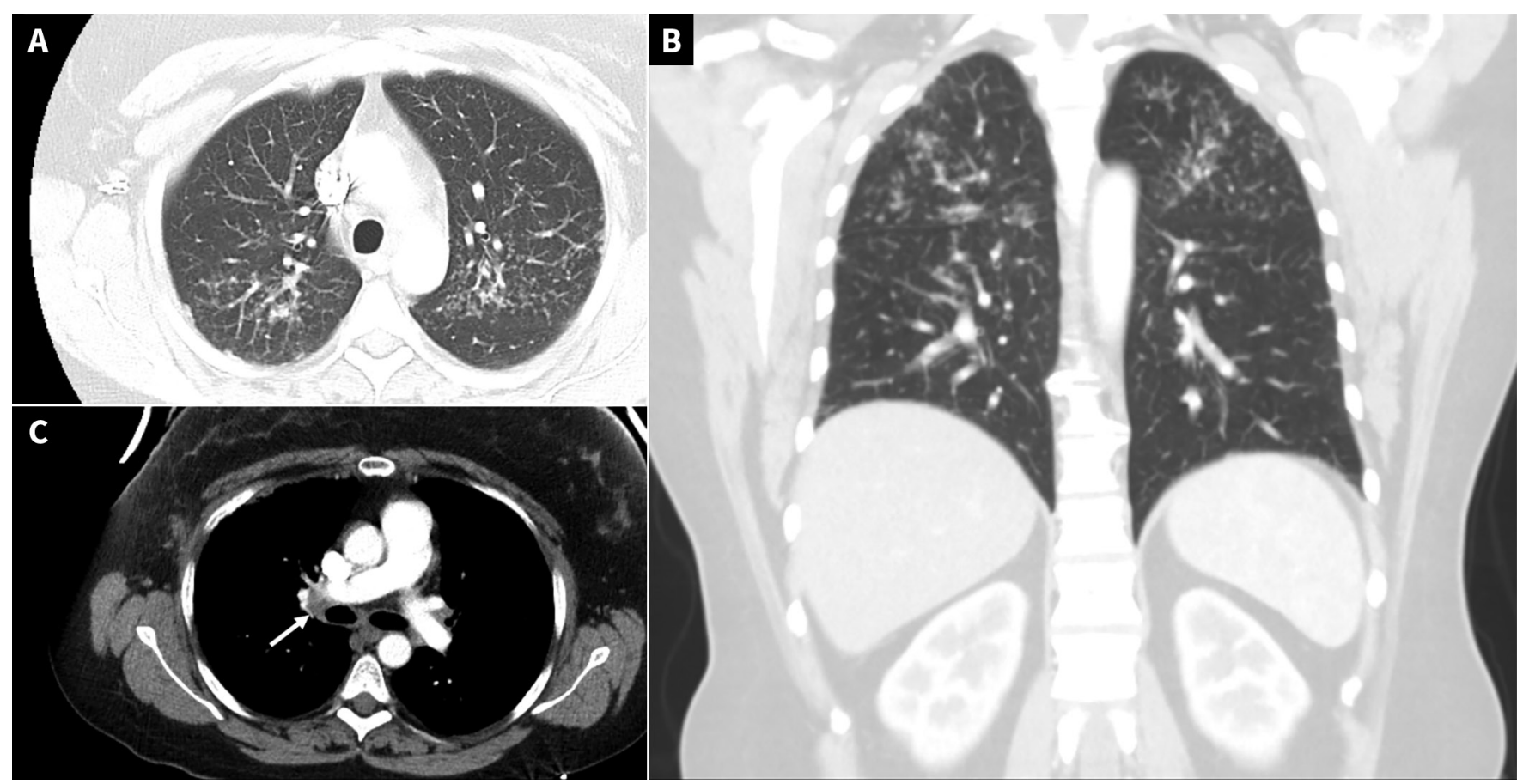

Figure 2: Axial (A) and coronal (B) computed tomography (CT) images of the chest, showing micronodular pulmonary nodules in a peribronchovascular perilymphatic distribution. (C) Axial CT image of the chest, showing a prominent right hilar lymph node with no calcifications (arrow).

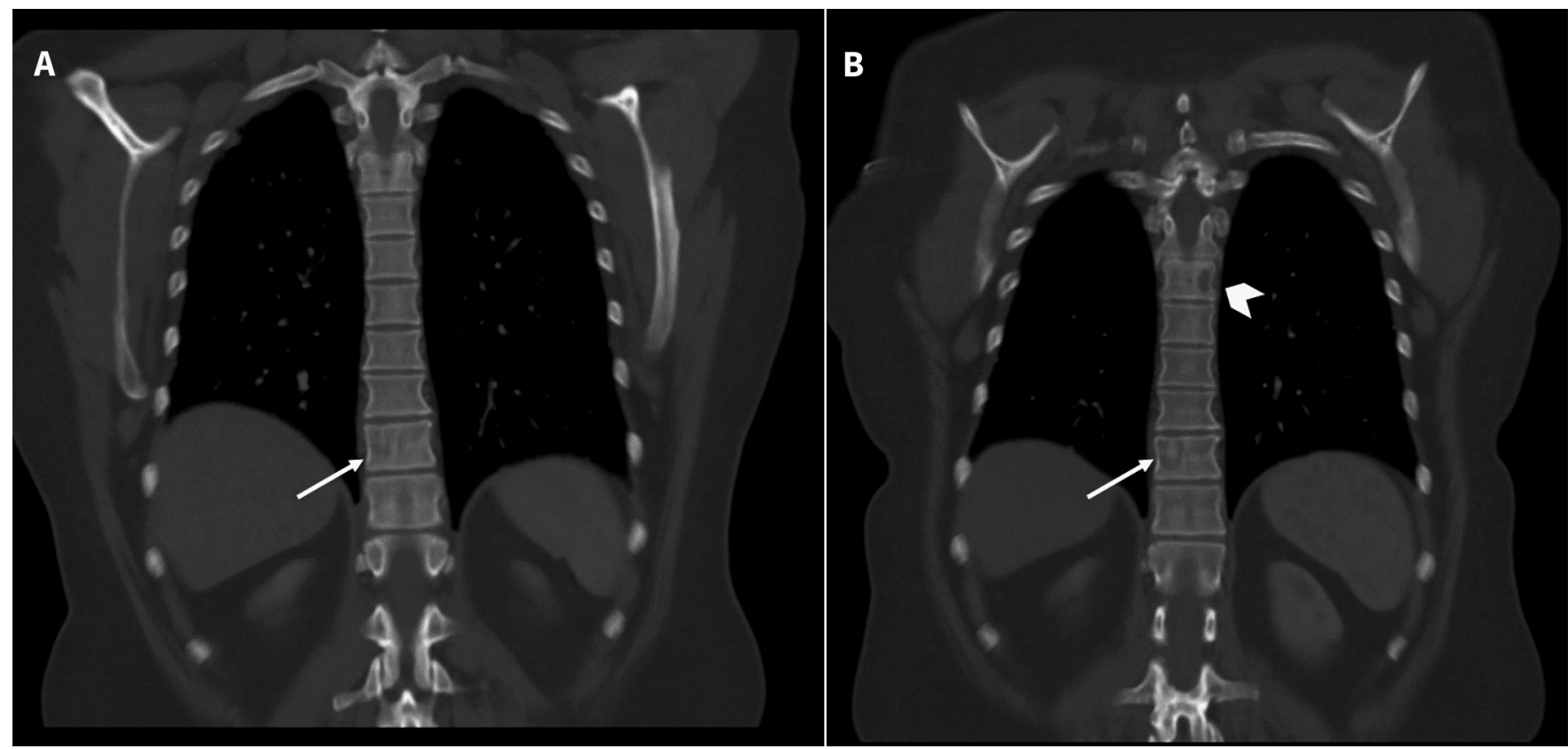

Figure 3: Coronal computed tomography images of the chest. (A) Possible small lucent lesion within the T10 vertebral body (arrow). (B) Image 2 years later shows a mixed lucent and sclerotic lesion about the right lateral aspect of the T10 vertebral body (arrow) and a lucent focus about the left lateral aspect of the T6 vertebral body (arrowhead).

On exclusion of alternative causes, a diagnosis of sarcoidosis was made. At the time, the patient had no respiratory symptoms or skin findings of sarcoidosis, and her calcium level was within the normal range.

The patient received adjuvant third-generation chemotherapy with a taxane and an anthracycline, followed by radiotherapy to the breast, supraclavicular fossa and axilla. She started endocrine therapy with tamoxifen, with a planned duration of 5-10 years. Repeat $\mathrm{CT}$ and bone scans done 4 months after the previous imaging showed stability of the pulmonary and bone findings (Figure 3A). Around this time, she developed a nonproductive cough, and treatment was started with an inhalational corticosteroid. 


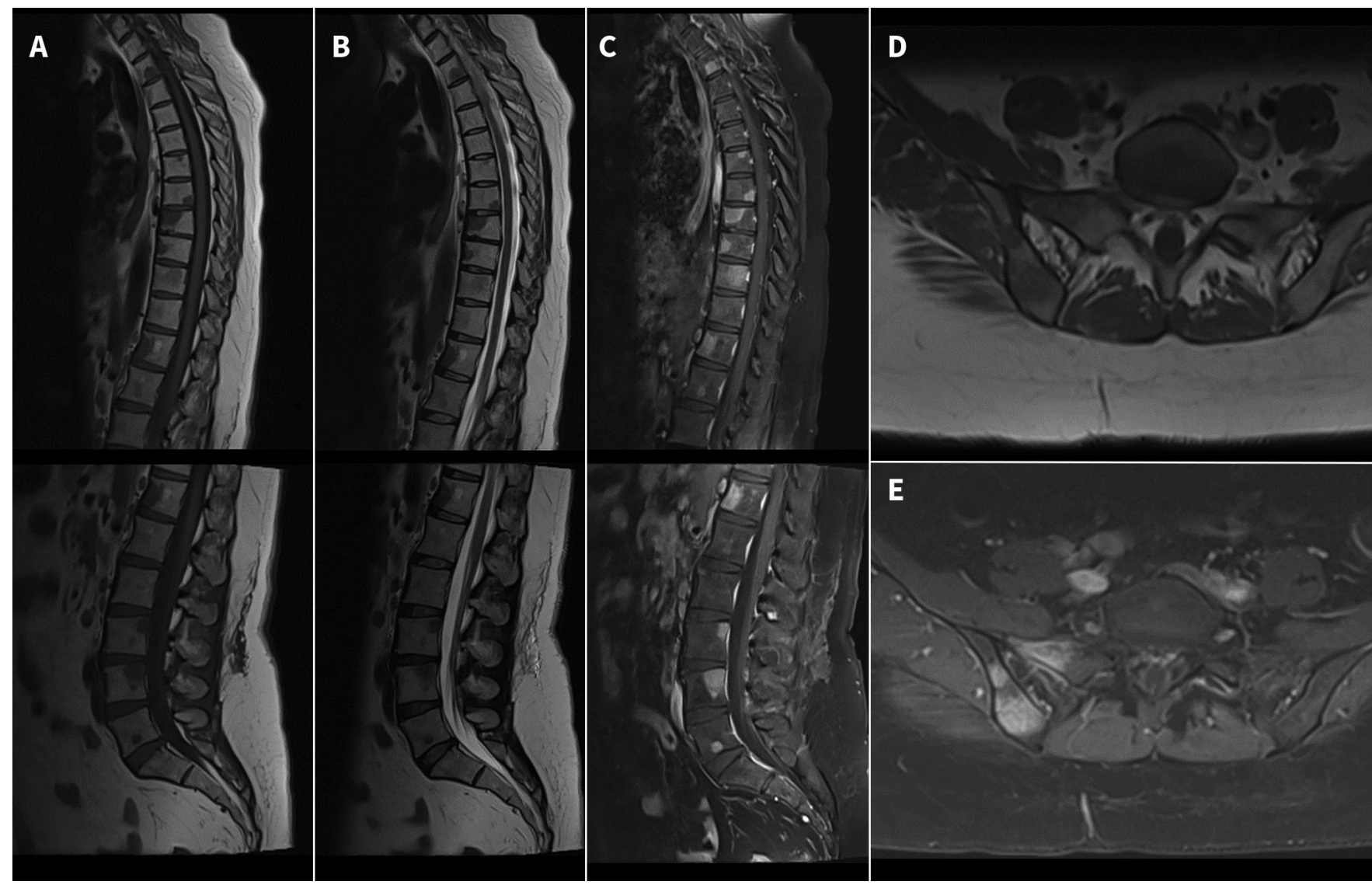

Figure 4: Magnetic resonance imaging views. (A) Sagittal $T_{1}$-weighted images of the thoracic and lumbar spine, showing multiple low-signal lesions in multiple vertebrae. (B) On sagittal $T_{2}$-weighted images, all of these lesions show low signal intensity. (C) Sagittal $T_{1}$-weighted fat-saturated postcontrast images, showing that all of these lesions enhanced after administration of gadolinium. (D) Axial $T_{1}$-weighted image of the sacrum, showing low-signal lesions within the right sacrum and right iliac bone. (E) Axial $T_{1}$-weighted fat-saturated image, showing that the sacral and iliac lesions enhanced after administration of gadolinium.

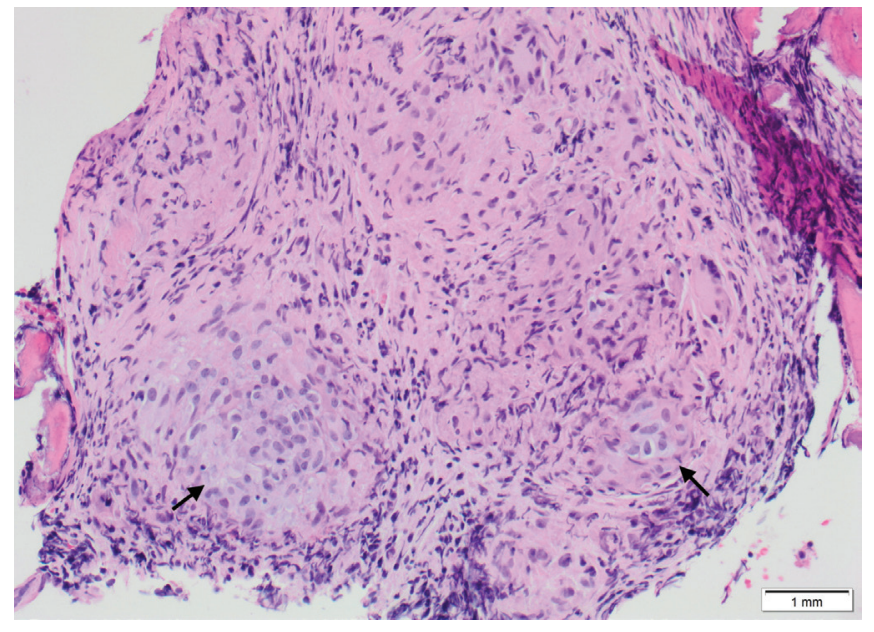

Figure 5: Biopsy specimen of the right iliac bone, showing multiple noncaseating granulomas (arrows) including multinucleated giant cells (hematoxylin-eosin, 20× magnification).

Two years after her breast cancer diagnosis, the patient noticed worsening of her cough. Chest-abdomen-pelvis CT showed increased demarcation of a lesion in T10 and new lucent lesions in T6 and L2 (Figure 3B). A bone scan showed no changes from the previous scan (Figure 1B). Magnetic resonance imaging (MRI) of the spine showed multiple osseous lesions, many of which were not visible on $\mathrm{CT}$ or bone scan (Figure 4). The imaging characteristics, including hypointensity on $T_{1}$ - and $T_{2}$-weighted sequences and postgadolinium enhancement, were suggestive of metastatic disease; however, there was no interval healing or flare response, as would be expected in the setting of metastatic lesions in a patient who had received treatment. Similarly, her alkaline phosphatase, cancer antigen 15-3 and calcium levels were within normal limits. Consequently, CT-guided core biopsy of a lesion in the right iliac bone was done to obtain a tissue diagnosis. The biopsy showed granulomas consistent with sarcoidosis (Figure 5).

The patient was treated with prednisone for management of sarcoidosis. At the time of writing, she remained free of breast cancer recurrence.

\section{Discussion}

Breast cancer is the most common cancer in women, with 1 in 8 Canadian women expected to be diagnosed with the disease during their lifetime. ${ }^{1}$ In 2019, there were an estimated 27200 new breast cancer diagnoses and 5100 breast cancer deaths in Canada. ${ }^{1}$ Prognosis and treatment options are heavily tied to TNM 
staging. Early-stage disease is treated with curative intent, involving surgery and possibly adjuvant chemotherapy, radiation and endocrine therapy. In the setting of metastatic disease, breast cancer is incurable, with only $27.4 \%$ of patients surviving 5 years. ${ }^{2}$ Treatment of stage IV breast cancer may include ongoing chemotherapy or endocrine therapy, which have associated adverse effects. It is therefore critical to assess stage accurately.

Bone is the most common site of metastasis, occurring in $65 \%-75 \%$ of patients with metastatic breast cancer. ${ }^{3}$ Assessment of bony metastatic disease is complicated by the nonspecific appearance of metastatic lesions and the lack of consensus on the optimal imaging modality for identifying bone metastases. ${ }^{4}$ Bone metastases from breast cancer are typically lytic; however, $15 \%-20 \%$ of women have blastic or mixed lesions. ${ }^{3}$ On MRI, lytic lesions are typically hypointense on $T_{1}$-weighted images and hyperintense on $T_{2}$-weighted images, whereas blastic lesions are typically hypointense on both $T_{1}$ - and $T_{2}$-weighted images. ${ }^{4}$ Many nonmalignant processes can mimic metastatic disease. In our case, given the patient's demographic characteristics, her history of malignant disease, and the appearance and location of the lesions, the differential diagnosis included metastatic disease, osseous sarcoidosis and multiple myeloma.

Sarcoidosis is a multisystem granulomatous disease of unknown cause. It is known to be associated with breast cancer; however, the true incidence of sarcoidosis in patients with breast cancer is unclear. Breast cancer has been shown to precede a diagnosis of sarcoidosis in $18 \%-24 \%$ of patients with sarcoidosis. ${ }^{5,6}$ The mechanism of this relation is unknown; however, it has been hypothesized that the development of sarcoidosis in the context of cancer may be the result of a Kveim response, whereby exposure to antigens resulting from the host immune response to cancer leads to the development of noncaseating granulomas. $^{7}$

Sarcoidosis is typically thought of as a pulmonary disease, but it can affect any organ in the body and involves bone in about $3 \%-13 \%$ of cases. ${ }^{8}$ Although many patients with bone involvement are asymptomatic, symptoms may include bony pain and swelling. ${ }^{9}$ The diagnosis of sarcoidosis requires compatible clinical features, biopsy findings of noncaseating granulomas and the exclusion of alternative causes of granulomatous inflammation. ${ }^{10}$ Similar to bone metastases from breast cancer, sarcoidosis of the bone typically has a lytic appearance; however, blastic or mixed lesions may be seen. ${ }^{8,9}$ The prognosis of sarcoidosis of the bone is quite favourable: up to one-third of patients never require systemic treatment, and 50\% experience spontaneous resolution within 2-5 years. ${ }^{10}$ For patients who require systemic treatment, corticosteroids are the first line of therapy; in refractory cases, methotrexate or other immunosuppressants can be considered. ${ }^{10}$

In our case, the patient's bone lesions were not consistent with the classic findings for either bone metastases or osseous sarcoidosis. Her lytic lesions were hypointense on both $T_{1}$ - and $T_{2}$-weighted images, and it was not possible to differentiate between these 2 disorders on imaging alone. This is consistent with a 2012 study showing how difficult it can be to differentiate reliably between these disorders, even for experienced MRI radiologists. ${ }^{8}$ Given the drastic difference in prognosis and management between bone metastases and osseous sarcoidosis, biopsy to obtain a tissue diagnosis may ultimately be required.

Although sarcoidosis of the bone is rare and is encountered much less frequently than skeletal metastases, it is important to consider it in the differential diagnosis in a patient with breast cancer, given the similar imaging characteristics and vastly different clinical course.

\section{References}

1. Canadian Cancer Statistics Advisory Committee. Canadian cancer statistics 2019. Toronto: Canadian Cancer Society; 2019. Available: www.cancer.ca/ /media/ cancer.ca/CW/cancer\%20information/cancer\%20101/Canadian\%20cancer\%20 statistics/Canadian-Cancer-Statistics-2019-EN.pdf?la=en (accessed 2019 Nov. 1).

2. Noone AM, Howlader N, Krapcho M, et al., eds. SEER cancer statistics review, 1975-2015. Bethesda (MD): National Cancer Institute; 2018. Available: https:// seer.cancer.gov/csr/1975_2015/(accessed 2019 Apr. 29).

3. Macedo F, Ladeira K, Pinho F, et al. Bone metastases: an overview. Oncol Rev 2017;11:321.

4. Cook GJR, Azad GK, Goh V. Imaging bone metastases in breast cancer: staging and response assessment. J Nucl Med 2016;57(Suppl 1):27S-33S.

5. Schweitzer MD, Salamo O, Holt G, et al. Sarcoidosis onset after breast cancer; a potential association. Eur J Intern Med 2017;44:e11-2.

6. Hunt BM, Vallières E, Buduhan G, et al. Sarcoidosis as a benign cause of lymphadenopathy in cancer patients. Am J Surg 2009;197:629-32, discussion 632.

7. Reich JM. Neoplasia in the etiology of sarcoidosis. Eur J Intern Med 2006;17:81-7.

8. Moore SL, Kransdorf MJ, Schweitzer ME, et al. Can sarcoidosis and metastatic bone lesions be reliably differentiated on routine MRI? AJR Am J Roentgenol 2012;198:1387-93.

9. Zhou Y, Lower EE, Li H, et al. Clinical characteristics of patients with bone sarcoidosis. Semin Arthritis Rheum 2017;47:143-8.

10. O’Regan A, Berman JS. Sarcoidosis. Ann Intern Med 2012;156:ITC5-1-15; quiz ITC5-16.

Competing interests: Christina Kim reports a research grant from Celgene, outside the submitted work. No other competing interests were declared.

This article has been peer reviewed.

The authors have obtained patient consent.

Affiliations: Max Rady College of Medicine (Li) and Department of Internal Medicine (Kim), Rady Faculty of Health Sciences, University of Manitoba; Sections of Adult Radiology (Stillwater) and Nuclear Medicine (Bryanton), Department of Radiology, University of Manitoba; Research Institute in Oncology and Hematology (Kim), CancerCare Manitoba, Winnipeg, Man.

Contributors: Henry Li and Christina Kim reviewed the patient's chart and drafted the manuscript. Laurence Stillwater and Mark Bryanton reviewed all imaging in detail and provided pictures. All of the authors revised the manuscript critically for important intellectual content, approved the final version to be published and agreed to be accountable for all aspects of the work.

Acknowledgement: The authors thank Dr. Ibrahim Aljada for providing the image for Figure 4.

Correspondence to: Henry Li, lih34525@myumanitoba.ca 